ESJ Natural/Life/Medical Sciences

\title{
Connaissances du Personnel Médical sur la Période des 1000 Premiers jours de vie à Lomé, Togo
}

\author{
D.A.E. Akolly \\ Service de Pédiatrie du CHU Sylvanus Olympio, de Lomé, \\ Université de Lomé, Togo \\ A.A. Ketevi
}

Service de Gynécologie et Obstétrique du CHU Sylvanus Olympio de Lomé,

Université de Lomé, Togo
E. Tsolenyanu
K.M. Guedenon
F. Agbeko
K.E. Djadou
A.D. Gbadoe

Service de Pédiatrie du CHU Sylvanus Olympio, de Lomé,

Université de Lomé, Togo

\section{Doi:10.19044/esj.2021.v17n10p137}

Submitted: 16 November 2020

Accepted: 12 February 2021

Published: 31 March 2021
Copyright 2021 Author(s)

Under Creative Commons BY-NC-ND

4.0 OPEN ACCESS

Cite As:

Akolly D.A.E., Ketevi A.A., Tsolenyanu E., Guedenon K.M., Agbeko F., Djadou K.E. \& Gbadoe A.D. (2021). Connaissances du Personnel Médical sur la Période des 1000 Premiers jours de vie à Lomé, Togo. European Scientific Journal, ESJ, 17(10), 137.

https://doi.org/10.19044/esj.2021.v17n10p137

\section{Resume}

Introduction: La période des 1000 premiers jours est celle qui va de la conception jusqu'au deuxième anniversaire de l'enfant. Cette période unique et critique pendant laquelle se met en place le potentiel physique, psychomoteur, intellectuel et cognitif conditionne la santé immédiate et future de l'enfant. Notre travail avait pour objectif d'évaluer les connaissances des médecins sur cette période clé. Méthode : Un questionnaire a été administré à un total de 71 médecins. Résultats : Près de la moitié $(49,30 \%)$ avaient déjà entendu parler du concept des 1000 premiers jours au moment de l'enquête. Le groupe pédiatre et médecins en cours de spécialisation en pédiatrie (soit respectivement 10/71 et 32/71) avaient une meilleure connaissance du concept 
$(\mathrm{p}=0,04)$ par rapport au groupe gynécologues et médecins en cours de spécialisation en gynécologie (soit respectivement $5 / 71$ et 28/71). Un médecin sur cinq ignorait les risques encourus à l'âge adulte lorsque la croissance fœtale n'était pas optimale. Trente médecins $(42,25 \%)$ estimaient leurs connaissances sur le microbiote intestinal insuffisantes ou médiocres. La moitié des médecins (50,70\%) affirmaient avoir abordé le thème des 1000 premiers jours avec leurs patients. Les connaissances des médecins spécialistes de la mère et de l'enfant au Togo sur le concept des 1000 premiers jours doivent être améliorées. Conclusion: Un renforcement général des compétences s'avère nécessaire pour espérer réduire le fardeau de toutes les maladies non transmissibles qui trouvent leur origine depuis la conception et pour lesquelles des actions efficaces peuvent encore être menées dans la petite enfance.

Mots-clés: 1000 premiers jours, médecins, Togo

\title{
Knowledge of Physicians on the Period of the first 1000 Days of Life in Lomé, Togo
}

\author{
D.A.E. Akolly \\ Service de Pédiatrie du CHU Sylvanus Olympio, de Lomé, \\ Université de Lomé, Togo
}

A.A. Ketevi

Service de Gynécologie et Obstétrique du CHU Sylvanus Olympio de Lomé, Université de Lomé, Togo

E. Tsolenyanu

K.M. Guedenon

F. Agbeko

K.E. Djadou

A.D. Gbadoe

Service de Pédiatrie du CHU Sylvanus Olympio, de Lomé, Université de Lomé, Togo

\begin{abstract}
Introduction: The first 1000 days is the period from conception to the child's second birthday. This unique and critical period during which the physical, psychomotor, intellectual, and cognitive potential is established conditions the immediate and future health of the child. This paper focuses on evaluating the knowledge of physicians during this key period. Method: A questionnaire was administered to a total of 71 physicians. Results: Nearly half $(49.30 \%)$ had already heard of the concept of the first 1000 days at the
\end{abstract}


time of the survey. The pediatrician and pediatric subspecialty group (10/71 and $32 / 71$ respectively) had a better knowledge of the concept $(\mathrm{p}=0.04)$ compared to the gynecologist and gynecology subspecialty group (5/71 and $28 / 71$ respectively). One in five physicians were unaware of the risks involved in adulthood when fetal growth was not optimal. Thirty physicians $(42.25 \%)$ considered their knowledge of the intestinal microbiota to be insufficient or poor. Half of the physicians $(50.70 \%)$ stated that they had discussed the first 1000 days with their patients. The knowledge of mother and child specialists in Togo on the concept of the first 1000 days needs to be improved. Conclusion: A general strengthening of skills is necessary to reduce the burden of all those noncommunicable diseases that originate from conception and for which effective actions can still be carried out in early childhood.

Keywords: First 1000 days, physicians, Togo

\section{Introduction}

L'implosion des maladies chroniques non transmissibles dans le monde et la nécessité d'une approche différente en matière de santé a obligé l'OMS à lancer le concept des 1000 premiers jours (Junien, 2011). La période des 1000 premiers jours est celle qui va de la conception jusqu'au deuxième anniversaire de l'enfant. Cette période unique et critique pendant laquelle se met en place le potentiel physique, psychomoteur, intellectuel et cognitif conditionne la santé immédiate et future de l'enfant. L'origine fotale des maladies métaboliques et cardiovasculaires de l'adulte est aujourd'hui clairement démontrée (Gluckman et al., 2008; Vickers et al., 2000 ; Gluckman et al., 2004 ; Darmaun, 2020 ; Roseboom et al., 2019 ; Godfrey et al., 2000). Ainsi, un faible poids de naissance suivi d'une prise de poids excessive dans la petite enfance entraine une augmentation du risque de maladies cardio-vasculaires comme l'hypertension artérielle (HTA) et le diabète de type 2 à l'âge adulte (Barker et al., 2002 ; Barker, 2004). Il est donc important que les apports nutritionnels soient optimaux au cours de cette période.

$\mathrm{Au}$ Togo, à notre connaissance, aucune étude n'a encore été menée pour évaluer l'état des connaissances sur ce concept au sein du personnel médical en charge des femmes enceintes et des jeunes enfants. Les médecins spécialistes et en cours de spécialisation de la mère et de l'enfant sont les acteurs de santé en première ligne pouvant avoir un impact décisif car, de toutes les actions menées au cours de cette période, va dépendre la vie future de l'enfant (Cunha et al., 2015). De ce fait, notre travail avait pour objectif d'évaluer les connaissances de ces professionnels. 


\section{Méthodologie}

Il s'agit d'une étude transversale descriptive menée du $1^{\text {er }}$ au 30 novembre 2019 au CHU Sylvanus Olympio de Lomé au Togo. Nous avons inclus dix pédiatres, cinq gynécologues, vingt-huit médecins en spécialisation en pédiatrie (douze en première année, cinq en deuxième année, quatre en troisième année et sept en quatrième année) et vingt-huit médecins en spécialisation en gynécologie (onze en première année, huit en deuxième année, deux en troisième année et sept en quatrième année), soit un total de 71 médecins. La collecte des données a été faite à partir d'un questionnaire préétabli. Les variables étudiées concernaient : la connaissance du concept des « 1000 premiers jours de vie », les connaissances sur les supplémentations indispensables chez la femme enceinte et sur l'alimentation du nouveau-né et du nourrisson jusqu'à deux ans. Les médecins ont été répartis en deux groupes pour l'analyse statistique; d'une part les pédiatres et les médecins en spécialisation en pédiatrie et d'autre part les gynécologues et les médecins en spécialisation en gynécologie. L'analyse statistique a été faite à l'aide du logiciel épi data version 3.1. Le seuil de signification de p était de 5\%.

\section{Résultats}

L'âge moyen des médecins ayant participé à l'enquête était de 34,08 $+/-5,6$ ans avec des extrêmes de 21 ans et 50 ans. Le sexe ratio H/F était de 1,05 .

Près de la moitié des enquêtés $(49,30 \%)$ avaient déjà entendu parler du concept des 1000 premiers jours, et ce par le biais d'internet, des enseignements post universitaires et des congrès médicaux. Quarante et un médecins $(57,74 \%)$ affirmaient connaitre et avaient pu donner la définition exacte de la période des 1000 premiers jours. Le concept des 1000 premiers jours était bien connu de $76,44 \%$ de médecins dans le groupe pédiatres et médecins en cours de spécialisation en pédiatrie par rapport au groupe gynécologues et médecins en cours de spécialisation en gynécologie $(51,85 \%)$ et le lien était significatif ( $\mathrm{p}=0,044)$. La majorité des médecins, respectivement $71,83 \%$ et $81,69 \%$, estimait qu'il s'agissait d'une période très importante dans la vie d'un individu et que la nutrition était très importante au cours de cette période.

Connaissances sur la grossesse et le développement foetal : Un médecin sur $5(20 \%)$ n'avait aucune connaissance sur la grossesse et le développement fœtal. Concernant les supplémentations indispensables en début de grossesse, $8,45 \%$ et $26,76 \%$ des enquêtés savait qu'il fallait supplémenter respectivement en iode et en calcium alors que, respectivement, $78,87 \%$ et $66,20 \%$ savaient qu'il fallait supplémenter en fer et en acide folique. 
Concernant les risques à long terme du retard de croissance intra-utérin (RCIU), environ le tiers des enquêtés (30,99\%) savaient qu'il y avait un risque ultérieur d'obésité et de diabète et la moitié $(50,70 \%)$ un risque de maladies cardio-vasculaires ; neuf médecins $(12,67 \%)$ pensaient qu'il n'y avait aucun risque.

Le faible poids de naissance (FPN) n'était pas lié à la mortalité infantile pour $14,28 \%$ des enquêtés et pour $29,58 \%$ il n'y avait aucun lien entre le FPN et la croissance future de l'enfant. Pour 36,62\%, le FPN n'augmente pas le risque de survenue de maladies coronariennes et $52,11 \%$ pensaient que la petite taille de naissance (PTN) n'augmentait pas le risque de survenue de maladies cardiovasculaires à l'âge adulte.

Pour 32,39\% des enquêtés, une croissance de rattrapage excessive n'augmentait pas le risque d'obésité ultérieure et $21,13 \%$ pensaient qu'une prise de poids excessive pendant l'enfance n'augmentait pas la mortalité d'origine cardio-vasculaire à l'âge adulte.

Connaissances sur l'alimentation: Trente médecins (42,25\%) estimaient leurs connaissances sur le microbiote intestinal insuffisantes ou médiocres. La majorité $(70,42 \%)$ savait que l'allaitement exclusif (AE) favorisait le développement optimal du microbiote alors que $23,94 \%$ pensait plutôt qu'une alimentation artificielle enrichie en pré et probiotique était préférable. Quarante-quatre médecins $(61,97 \%)$ savaient que l'allaitement maternel permettait de diminuer le risque de maladies allergiques.

La majorité des médecins $(85,92 \%)$ savaient que l'âge idéal pour démarrer la diversification alimentaire était de six mois mais le tiers $(32,39 \%)$ estimait qu'il fallait attendre 12 mois pour introduire les œufs. Un tiers soit $32,39 \%$ savait que l'introduction précoce des aliments solides augmentait le risque d'obésité alors que $7 \%$ pensait que cette pratique permettrait au contraire d'éviter la survenue de l'obésité. Un peu plus des deux tiers (70,42\%) savaient que l'exposition répétée à un nouvel aliment au cours de la diversification pouvait conduire à son acceptation alors que $19,72 \%$ pensaient que cette pratique pouvait conduire au contraire à son refus. Quarante-cinq médecins soit $63,38 \%$ savaient que l'exposition visuelle antérieure favorisait l'acceptation des aliments par l'enfant alors que $11,27 \%$ pensaient que cette pratique conduirait plutôt au refus par l'enfant. Pour $15,49 \%$ des praticiens, la texture de l'aliment n'avait aucune incidence au cours de la diversification. Les connaissances du groupe pédiatres et médecins en spécialisation de pédiatrie sur la diversification étaient meilleures par rapport au groupe gynécologues et médecins en spécialisation en gynécologie $(p=0,03)$. Seulement $19,72 \%$ des médecins savaient que le rebond d'adiposité normal se produisait autour de 6 ans et parmi les facteurs favorisant la survenue d'un rebond d'adiposité précoce (RAP) seulement un médecin sur 4 
$(25,35 \%)$ savait qu'un apport protéique excessif pouvait être en cause. Plus de 2 médecins sur 3 interrogés $(73,24 \%)$ savaient que des interventions diététiques pouvaient permettre de corriger un rebond d'adiposité précoce (RAP).

Près de la moitié des médecins $(45 \%)$ savaient que la carence en fer pouvait être responsable de troubles cognitifs chez l'enfant alors que pour $11,27 \%$ des médecins, la carence en fer n'avait aucune conséquence sur l'enfant.

La moitié des médecins $(50,70 \%)$ affirmaient avoir abordé le thème des 1000 premiers jours avec leurs patients ; et ceci à leur propre initiative dans $35,21 \%$ et en réponse à des questions dans $39,43 \%$.

\section{Commentaires}

Au début des années 1980, BARKER avait démontré que le risque de décès par maladie coronarienne à l'âge adulte était corrélé au poids du sujet à la naissance, avec un risque de transmission aux générations suivantes (Barker et al., 2005 ; Roseboom et al., 2001).

Un médecin sur cinq ignorait les risques encourus à l'âge adulte lorsque la croissance foetale n'était pas optimale alors qu'il est aujourd'hui clairement démontré qu'une restriction nutritionnelle au cours de la vie intrautérine entraîne des modifications permanentes au niveau métabolique qui vont persister jusqu'à l'âge adulte et seront à l'origine des maladies comme l'HTA, les maladies coronariennes, le diabète de type 2 et l'obésité (Kajantie et al., 2008 ; Osmond et al., 2000 ; Whincup et al., 2008 ; Osmond et al., 1993 ; Erickson et al., 2001).

Dans 30 à 50\% des cas, les risques liés au RCIU, au FPN, à la PTN de même qu'au rattrapage excessif dans la petite enfance étaient mal connus. Une malnutrition maternelle pendant la grossesse, un placenta de faible poids et une vie dans des conditions socio-économiques défavorables après la naissance augmentent le risque d'HTA à l'âge adulte (Barker, 2007). Un bébé conçu en période de famine et né en période normale aura un poids normal à l'âge adulte mais aura à son tour des bébés de FPN: c'est l'effet intergénérationnel. La croissance des filles influence donc la survenue de maladies chroniques dans la prochaine génération (Roseboom et al., 2019; Godfrey et al., 2000 ; Barker, 1997). La PTN est la conséquence de carences en fin de grossesse ; en effet la déviation du flux sanguin vers le cerveau qui en résulte pénalise le foie expliquant la baisse du périmètre ombilical constatée à la naissance qui traduit une diminution de la taille du foie. Le foie intervient dans le métabolisme du cholestérol et ces perturbations métaboliques expliquent la survenue des maladies coronariennes à l'âge adulte. La croissance intra-utérine et pendant la petite enfance est un facteur de risque de développement d'un groupe de maladies chroniques comme les maladies 
coronariennes, les AVC, le diabète de type 2 et l'HTA. La malnutrition intrautérine change la plasticité des organes: c'est le phénomène de programmation. Si un enfant présente un RCIU et un retard de croissance dans l'enfance suivi d'un rattrapage excessif, cela entraîne des maladies coronariennes et le diabète de type 2 alors que l'absence de rattrapage favorise plutôt la survenue des AVC (Erickson et al., 2001 ; Barker et al., 2009; Embleton et al., 2016). Les hommes ayant eu un faible poids à la naissance ont une augmentation de la mortalité d'origine cardiovasculaire à l'âge adulte (Kajantie et al., 2005). La croissance post natale de ces FPN devrait être surveillée car une croissance de rattrapage excessive entraîne un risque ultérieur d'obésité. Le RCIU, le FPN et la PTN entrainent des maladies cardiovasculaires, le diabète de type 2 et une hypercholestérolémie dont la prévention passe par l'assurance d'un équilibre nutritionnel au cours de la grossesse (Barker, 2000).

En début de grossesse, il est indispensable de supplémenter en acide folique, en iode et en calcium. La carence en fer au cours de la grossesse augmente le risque de syndrome métabolique à long terme chez le fœtus. Si la supplémentation en fer et acide folique est bien connue, la supplémentation en iode et en calcium qui sont des oligo-éléments également indispensables sont peu connus des médecins. L'iode a un rôle déterminant dans le développement cérébral. La poursuite de la supplémentation en acide folique tout le long de la grossesse permet d'améliorer le développement cognitif des enfants. La carence en fer précoce entraine des troubles cognitifs qui peuvent persister à l'âge adulte (Devakumar et al., 2016 ; McNulty et al., 2019).

Près de la moitié des médecins estimaient leurs connaissances sur le microbiote insuffisantes même si la majorité savait que l'allaitement exclusif pendant les 6 premiers mois de vie favorisait son développement optimal. Le microbiote se développe pendant les deux premières années de vie et a des fonctions nutritionnelle, physiologique, immunitaire et métabolique. Dans un tiers des cas, les connaissances sur la conduite de l'alimentation du nourrisson étaient insuffisantes. L'allaitement exclusif pendant les 6 premiers mois et l'exposition visuelle antérieure et répétée à une variété de saveurs dès le début de la diversification favorise l'acceptation des aliments par les enfants et conduit à une croissance optimale (Maier et al., 2007).

\section{Conclusion}

Ce travail a permis d'évaluer les connaissances des médecins au Togo sur le concept des 1000 premiers jours de vie. Même si les deux tiers des médecins enquêtés connaissaient le concept, un médecin sur cinq n'avaient aucune connaissance sur le déroulement optimal de la grossesse. Les connaissances sur l'alimentation étaient bonnes dans les deux tiers des cas. Un renforcement général des compétences s'avère nécessaire pour espérer réduire 
le fardeau de toutes ces maladies non transmissibles qui trouvent leur origine depuis la conception et pour lesquelles des actions efficaces peuvent encore être menées dans la petite enfance. En matière de santé publique, il serait plus rentable d'investir sur la période des 1000 premiers jours. Il s'agit à la fois d'une période de vulnérabilité mais aussi d'opportunité à saisir pour améliorer la santé des générations actuelles et futures.

\section{References:}

1. Barker, DJP. (1997). Maternal nutrition, fetal nutrition, and disease in later life. Nutrition;13(9).

2. Barker, DJP. (2000). In utero programming of cardiovascular disease. Theriogenology.; 53:555-74.

3. Barker, DJP., Eriksson, JG., Forsén, T., \& Osmond, C. (2002). Fetal origins of adult disease: strength of effects and biological basis. Int. J. Epidemiol; 31 (6):1235-1239.

4. Barker, JP. (2004). The developmental origins of adult disease. J Am Coll Nutr ; 23 (6) : 588S-595S.

5. Barker, DJ., Osmond, C., Forsén, TJ., Kajantie, E., \& Eriksson, JG. (2005). Trajectories of Growth among Children Who Have Coronary Events as Adults. N Engl J Med;353(17):1802-9.

6. Barker, DJP., Osmond, C., Forsen, TJ., Kajantie, E., \& Eriksson, JG. (2007). Maternal and social origins of hypertension. Hypertension;50:565-571.

7. Barker, DJP., Osmond, C., Kajantie, E., \& Eriksson, JG. (2009). Growth and chronic disease: findings in the Helsinki Birth Cohort. Ann. Hum. Biol; 36(5): 445-58.

8. Cunha, AJLA., Leite, AJM., \& de Almeida, IS. (2015). The pediatrician's role in the first thousand days of the child: The pursuit of healthy nutrition and development. Jornal de Pediatria;S44-51.

9. Darmaun, D. (2020). La nutrition des mille premiers jours : quels enjeux ? Nutr clin métab; https//doi.org/10.1016/j.nupar.2020.04.004

10. Devakumar, D., Fall, CHD., Sachdev, HS., Margetts, BM., Osmond, C., Wells, JCK. et al. (2016). Maternal antenatal multiple micronutrient supplementation for long-term health benefits in children: a systematic review and meta-analysis. BMC;14(1):90.

11. Embleton, ND., Korada, M., Wood, CL., Pearce, MS., Swamy, R., \& Cheetham, TD. (2016). Catchup growth and metabolic outcomes in adolescents born preterm. Arch Dis Child;101:1026-31.

12. Eriksson, JG., Forsen, T., Tuomilehto, J., Osmond, C., \& Barker, DJP. (2001). Early growth and coronary heart disease in later life: longitudinal study. BMJ; 322 (7292), 949-953. 
13. Gluckman, PD., \& Hanson, MA. (2004). Developmental origins of disease paradigm: a mechanistic and evolutionary perspective. Pediatr Res; $56: 311-7$

14. Gluckman, PD., Hanson, MA., Cooper, C., \& Thornburg, KL. (2008). Effect of in utero and early life conditions on adult health and disease. N Engl J Med ;359(1):61-73. doi: 10.1056/NEJMra0708473.

15. Godfrey, KM., \& Barker, DJ. (2000). Fetal nutrition and adult disease. Am J Clin Nutr;71(Suppl.):1344S-52S.

16. Junien, C., \& Siméoni, U. (2011). L'initiative des 1000 jours de l'OMS et l'origine développementale de la santé et des maladies (DOHaD). Document en ligne www.sf-dohad.fr consulté le 18 mai 2020 à 14h.

17. Kajantie, E., Barker, DJP., Osmond, C., Forsen, T., \& Eriksson, JG. (2008). Growth before 2 years of age and serum lipids 60 years later: the Helsinki birth cohort study. Int. J. Epidemiol;37:280 -289.

18. Kajantie, E., Osmond, C., Barker, DJP., Forsen, T., Phillips, DIW., \& Eriksson, JG. (2005). Size at birth as a predictor of mortality in adulthood: a follow-up of 350000 person-years Int. J. Epidemiol;34:655-63.

19. Maier, A., Chabanet, C., Schaal, B., \& Issanchou, S. (2007). Effects of repeated exposure on acceptance of initially disliked vegetables in 7month old infants. Food Qual Prefer;18(8):1023-32.

20. McNulty, H., Rollins, M., Cassidy, T., Caffrey, A., Marshall, B., Dornan, J. et al. (2019). Effect of continued folic acid supplementation beyond the first trimester of pregnancy on cognitive performance in the child: a follow-up study from a randomized controlled trial (FASSTT Offspring Trial). BMC Medicine; 17: 196.

21. Osmond, C., \& Barker, DJP. (2000). Fetal, infant, and childhood growth are predictors of coronary heart disease, diabetes, and hypertension in adult men and women. Environ Health Perspect ;108(3):545-553.

22. Osmond, C., Barker, DJ., Winter, PD., Fall, CH., \& Simmonds, SJ. (1993). Early growth and death from cardiovascular disease in women. BMJ.; 307 (6918): 1519-1524.

23. Roseboom, TJ. (2019). Epidemiological evidence for the developmental origins of health and disease: effects of prenatal undernutrition in humans. J Endocrinol;242:T135-44.

24. Roseboom, TJ., Van der Meulen, JHP., Ravelli, ACJ., Osmond, C., Barker, DJP., Bleker, OP. (2001). Effects of prenatal exposure to the dutch famine on adult disease in later life: an overview. Twin Research. ; 4 (5) : 293- 298.

25. Vickers, MH., Breier, BH., Cutfield, WS., Hofman, PL., \& Gluckman, PD. (2000). Fetal origins of hyperphagia, obesity, and hypertension 
and postnatal amplification by hypercaloric nutrition. Am J Physiol Endocrinol Metab;279:E83-E87.

26. Whincup, PH., Kaye, SJ., Owen, CG., Huxley, R., Cook, DG., \& Anazawa, S. (2008). Birth weight and risk of type 2 diabetes: a systematic review. JAMA;300(24):2886-2897. 